\title{
Experimental and numerical study of the evolution of stored and dissipated energies in a medium carbon steel under cyclic loading
}

\author{
C. Mareau ${ }^{\mathrm{a}}$, D. Cuillerier ${ }^{\mathrm{a}}$, F. Morel ${ }^{\mathrm{a}}$ \\ a Arts et Métiers ParisTech, CER d'Angers, LAMPA, 2 bd du Ronceray, 49035 Angers Cedex 1, \\ France
}

\begin{abstract}
To obtain robust estimations of the fatigue limit from energy-based fatigue criteria, constitutive laws must include a correct description of the energy balance when modeling the cyclic behavior. The present paper aims at providing a better understanding of the evolution of the energy balance at both microscopic and macroscopic scales in a medium carbon steel. First, an experimental procedure is used to estimate the amount of energy which is either stored in the material or dissipated into heat at a macroscopic scale. The energy balance is observed to be very dependent on the stress amplitude and the number of loading cycles. A model is then developed to investigate the energy balance at a microscopic scale. From the simulation results, both the stored energy and dissipated energy fields are found to be strongly scattered. The dispersion is mostly explained by the crystallographic orientation distribution and the two-phased microstructure.
\end{abstract}

Key words: stored energy, dissipated energy, steel, crystal plasticity

\section{Introduction}

In-service loading conditions usually generate complex cyclic stress states. As such, the choice of an appropriate multiaxial fatigue criterion plays a crucial role in obtaining correct fatigue predictions. In the case of high cycle fatigue, the observation of the stabilized behavior is generally required to build either stress-based criteria (Crossland, 1956; Dang Van, 1973; Morel, 2000; Papadopoulos, 1994; Sines, 1959) or energy-based criteria

Email address: charles.mareau@ensam.eu (C. Mareau). 
(Banvillet et al., 2003; Ellyin, 1974; Leis, 1977; Palin-Luc and Lasserre, 1998). The different energy-based approaches can be classified according to the kind of energy which is assumed to drive the fatigue process. Indeed, the total strain energy is not entirely dissipated into heat since a fraction is stored in the material. While the link between fatigue damage and dissipated energy remains unclear (Chrysochoos et al., 2008), the interest for stored energy approaches is motivated by the correlation that exists between stored energy and fatigue damage accumulation (Warren and Wei, 2010). More specifically, energy storage and fatigue damage are both related to the behavior of dislocations. Energy storage corresponds to an increase of the free energy associated with the multiplication of defects (e.g. dislocations, vacancies) while fatigue damage often results from the evolution of the dislocation substructure (Polák, 2007). Consequently, constitutive laws must include a correct description of the energy balance to obtain robust estimations of the fatigue life under multiaxial loading conditions from energetic approaches.

In order to model the cyclic behavior, different solutions have been proposed. On the one hand, macroscopic plasticity models showed their ability to reproduce several features of the cyclic behavior such as the Bauschinger effect or cyclic hardening and softening (Armstrong and Frederick, 1966; Marquis, 1979). On the other hand, polycrystalline plasticity models have been developed to include the influence of microstructural heterogeneities (Berveiller and Zaoui, 1979; Hill, 1965; Hutchinson, 1976; Lebensohn and Tomé, 1993; Paquin, 2001). Indeed, polycrystalline plasticity models are able to account for the anisotropy of single crystal properties which is known to reinforce the heterogeneous aspect of both the elastic-plastic deformation and the dissipative behavior of polycrystalline aggregates (Mareau et al., 2012; Roters et al., 2010). Also, to deal with intragranular heterogeneities, the computation of the stored energy associated with the internal stress field surrounding dislocation structures has been performed from discrete dislocation dynamic simulations (Déprés et al., 2006; Mura, 1994; Zehnder, 1991). Discrete dislocation dynamic simulations are however limited to small volume elements.

In the present paper, it is proposed to study the evolution of the energy balance at both macroscopic and microscopic scales. The studied material is a medium carbon steel which is submitted to a uniaxial cyclic loading with different stress amplitudes. First, the experimental procedure which is used to estimate the evolution of stored and dissipated energies at a macroscopic scale is presented. Then, to gain insight into the energy balance at a microscopic scale, a polycrystalline model is proposed. Finally, the experimental estimations of stored and dissipated energies are discussed and compared to the results obtained from the polycrystalline model.

\section{Experimental procedure}

\subsection{Material description}

The material which is studied in the present work is a medium carbon steel (C35) whose chemical composition and mechanical and thermal properties are given in tables 1 and 2. The material does not exhibit a significant texture and it is made of spherical grains whose size is about $12 \mu \mathrm{m}$. The material is two-phased (ferrite and cementite) with a volume fraction of proeutectoid ferrite of about $55 \%$ while the volume fraction of pearlite is about $45 \%$. 
Table 1

\begin{tabular}{cccccc}
\hline \hline $\mathrm{C}$ & $\mathrm{Si}$ & $\mathrm{Mn}$ & $\mathrm{P}$ & $\mathrm{Cr}$ & $\mathrm{Fe}$ \\
$0.38 \%$ & $0.23 \%$ & $0.86 \%$ & $0.02 \%$ & $0.04 \%$ & balance \\
\hline \hline
\end{tabular}

Chemical composition (wt\%) of the C35 steel.

\begin{tabular}{cccccc}
\hline \hline$\sigma_{y}(\mathrm{MPa})$ & $U T S(\mathrm{MPa})$ & $E(\mathrm{MPa})$ & $A \%(\%)$ & $\rho\left(\mathrm{kg} \cdot \mathrm{m}^{-3}\right)$ & $C\left(\mathrm{~J} \cdot \mathrm{kg}^{-1} \cdot \mathrm{K}^{-1}\right)$ \\
430 & 650 & 205000 & 18 & 7800 & 450 \\
\hline \hline Table 2 & & &
\end{tabular}

Mechanical and thermal properties of the C35 steel: yield stress $\left(\sigma_{y}\right)$, ultimate tensile strength (UTS), Young modulus $(E)$, elongation at break $(A \%)$, mass density $(\rho)$ and specific heat $(C)$.

\subsection{Experimental setup}

The cyclic tests have been performed with an INSTRON 8850 fatigue testing machine on specimens with a tubular geometry. The specimen geometry is detailed in figure 1 . Each specimen has been submitted to a load-controlled cyclic loading with a constant load ratio $R=\Sigma_{\min } / \Sigma_{\max }$ such that the applied macroscopic stress is in the form:

$$
\Sigma=\Sigma_{m}+\Sigma_{a} \sin \left(2 \pi f_{l} t\right)
$$

where $\Sigma_{m}, \Sigma_{a}$ and $f_{l}$ are respectively the mean stress, the stress amplitude and the loading frequency $(20 \mathrm{~Hz}$ in the present work). The experimental data consist of:

- the macroscopic stress $\Sigma$ which is determined from the applied force,

- the macroscopic strain $E$ which is obtained thanks to an extensometer that is attached to the specimen,

- and the temperature $T$ which is monitored around the specimen center with a $T$ type thermocouple.

\subsection{Energy balance}

The determination of the amount of energy which is either stored in the material or dissipated into heat during cyclic tests is achieved by adopting the thermomechanical framework that was first proposed by Louche and Chrysochoos (2001) and modified by Boulanger et al. (2004) to account for the specific features of high cycle fatigue. Because of the low temperature elevations which are generally observed during our fatigue tests (i.e. $<20 \mathrm{~K}$ ), no microstructural evolution such as recrystallization or phase transformation is likely to occur so the contribution of the internal coupling source is neglected. Therefore, only the thermoelastic and dissipation sources are considered in the heat diffusion equation:

$$
\rho C \dot{\theta}-k \Delta \theta=D_{1}+S_{t h e}
$$

$C$ is the specific heat of the material, $\rho$ is the mass density, $k$ is the thermal conductivity (which is assumed to be isotropic), $\Delta$ is the Laplace operator, $\theta=T-T_{0}$ is the temperature elevation, which is defined as the difference between the absolute temperature $T$ and the equilibrium temperature $T_{0}$ and $D_{1}$ (respectively $S_{\text {the }}$ ) is the macroscopic dissipation source (respectively macroscopic thermoelastic source). In the above equation, because of the high thermal conductivity which is generally observed for steel, the temperature is quite homogeneous around the thermocouple so $\theta$ is treated as the volume average 


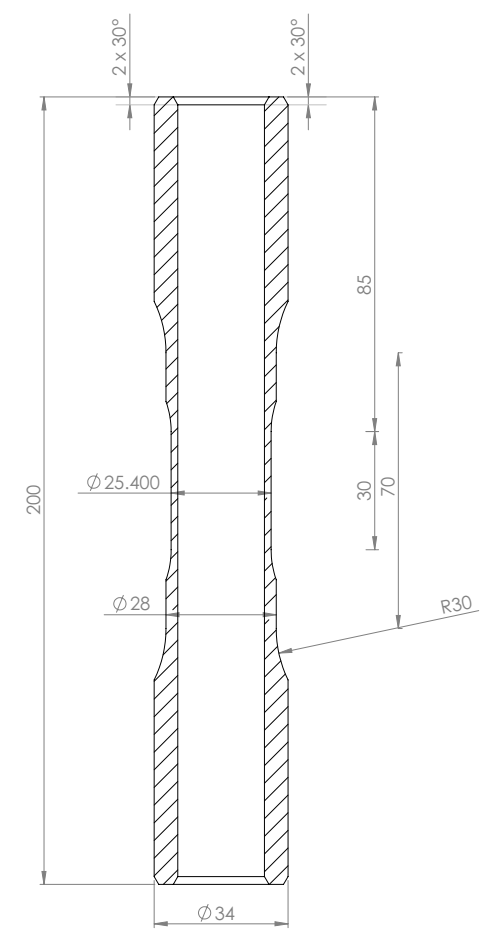

Fig. 1. Tubular specimen geometry.

temperature elevation around the specimen center. Also, due to low temperature gradients and displacement velocities, the convective terms associated with the particular time derivative of the temperature field are neglected (Boulanger et al., 2004).

According to Mareau et al. (2009), since the thermocouple response time is high in comparison with the loading period $t_{l}=1 / f_{l}$, it does not enable to record the instantaneous value of the temperature but only its average value per cycle determined over numerous loading cycles. Consequently, the above form of the heat diffusion equation can be further simplified if the temperature which is given by the thermocouple is considered to be equal to the time-averaged temperature determined over one loading cycle. Thus, by denoting $\bar{z}$ the average of $z(t)$ over one loading cycle, the heat diffusion equation can be rewritten in the following form:

$$
\rho C \overline{\dot{\theta}}-\overline{k \Delta \theta}=\bar{D}_{1}+\bar{S}_{\text {the }}
$$

The final form of the heat diffusion equation is obtained if the following assumptions are made:

- the sum of the thermoelastic source over one loading cycle is neglected $\left(\bar{S}_{\text {the }} \approx 0\right)$ since the volume element is close to thermal equilibrium (i.e. $\theta<<T_{0}$ ) (Boulanger et al., 2004),

- the heat losses between the specimen center and its environment are assumed to be quadratic with respect to the temperature elevation (i.e. $\overline{k \Delta \theta}=-\bar{\theta} / a-\bar{\theta}^{2} / b$ ). $a$ and $b$ are some constants characterizing heat losses that depend on both testing conditions and material properties. 
The average dissipation source $\bar{D}_{1}$ is thus estimated from temperature measurements from the following relation:

$$
\rho C \overline{\dot{\theta}}+\frac{\bar{\theta}}{a}+\frac{\bar{\theta}^{2}}{b}=\bar{D}_{1}
$$

The power $P_{\text {ext }}$ which is developed by external forces is given by:

$$
P_{e x t}=\Sigma: \dot{E}
$$

Adopting small deformation theory, the macroscopic strain is split into elastic $\left(E_{e}\right)$ and plastic $\left(E_{p}\right)$ parts:

$$
E=E_{e}+E_{p}
$$

Because of the linear relation between the stress tensor $\Sigma$ and the elastic strain tensor $E_{e}$, when relation (5) is averaged over one loading cycle, one obtains:

$$
\bar{P}_{e x t}=\overline{\Sigma: \dot{E}}=\overline{\Sigma: \dot{E}_{p}}
$$

Consequently, the average power of external forces per cycle $\bar{P}_{\text {ext }}$ can be obtained from experimental stress and strain data from equation (7). According to Lemaitre et al. (2009), the stored energy evolution rate $P_{s}$ is defined as the difference between the plastic work rate $\Sigma: \dot{E}_{p}$ and the dissipation source $D_{1}$. Thus, the average stored energy evolution rate per cycle is given by:

$$
\bar{P}_{s}=\bar{P}_{e x t}-\bar{D}_{1}
$$

Since $\bar{P}_{\text {ext }}$ and $\bar{D}_{1}$ are determined from equations (4) and (7), the difference between $\bar{P}_{e x t}$ and $\bar{D}_{1}$ provides an estimation of the average stored energy evolution rate per cycle.

\section{Model description}

\subsection{Homogenization scheme}

Let us consider a volume element $V$ which is representative of the studied material. The volume element is submitted to periodic boundary conditions such that finding the local strain rate $\dot{\varepsilon}$ and spin rate $\dot{\omega}$ at point $x$ as a function of the prescribed macroscopic strain rate $\dot{E}$ and spin rate $\dot{\Omega}$ consists of solving the following integral equations:

$$
\begin{aligned}
& \dot{\varepsilon}(x)=\dot{E}-\int_{V} \Gamma_{\text {sym }}^{C^{0}}\left(x-x^{\prime}\right): \dot{\tau}\left(x^{\prime}\right) d x^{\prime} \\
& \dot{\omega}(x)=\dot{\Omega}-\int_{V} \Gamma_{\text {skew }}^{C^{0}}\left(x-x^{\prime}\right): \dot{\tau}\left(x^{\prime}\right) d x^{\prime}
\end{aligned}
$$

$\Gamma_{\text {sym }}^{C^{0}}$ and $\Gamma_{\text {skew }}^{C^{0}}$ are the symmetric and skew-symmetric parts of the periodic Green operator associated with the reference elastic medium with elastic stiffness $C^{0}$ and $\dot{\tau}$ is the polarization rate field defined at point $x$ by:

$$
\dot{\tau}(x)=\dot{\sigma}(x)-C^{0}: \dot{\varepsilon}(x)
$$

In order to determine the local fields $\dot{\varepsilon}$ and $\dot{\omega}$, the FFT approach, which has been proposed by Moulinec and Suquet (1998) for heterogeneous materials with an elastic or an elastoplastic behavior and extended to the elasto-viscoplastic case by Lebensohn et al. (2012), 
is used. When the Fourier transform (denoted by $F$ ) is applied to the integral equations (9) and (10), the convolution product is transformed into a tensor product such that:

$$
\begin{gathered}
F(\dot{\varepsilon})(\xi)= \begin{cases}\dot{E} & \text { if } \xi=0 \\
-F\left(\Gamma_{\text {sym }}^{C^{0}}\right)(\xi): F(\dot{\tau})(\xi) & \text { otherwise }\end{cases} \\
F(\dot{\omega})(\xi)= \begin{cases}\dot{\Omega} & \text { if } \xi=0 \\
-F\left(\Gamma_{\text {skew }}^{C^{0}}\right)(\xi): F(\dot{\tau})(\xi) & \text { otherwise }\end{cases}
\end{gathered}
$$

If the representative volume element is discretized into voxels, an approximate solution of the problem is obtained by applying the fast Fourier transform to the integral equations (9) and (10). Indeed, since the modified Green operator is explicitly known in the frequency domain (Mura, 1987), the convolution product is evaluated in the frequency domain at a low computational cost and the inverse Fourier transform is performed to express the strain rate field in the original space domain. However, because the polarization rate field is a function of the unknown strain rate and stress rate fields, the problem needs to be solved iteratively. Though different advanced numerical resolution schemes have been proposed (Eyre and Milton, 1999; Michel et al., 2001; Zeman et al., 2010), the basic scheme from Moulinec and Suquet (1998) is used in the present work.

Adopting small deformation theory, both the strain rate $\dot{\varepsilon}$ and the spin rate $\dot{\omega}$ at a given point $x$ of the volume element are decomposed into elastic and plastic parts:

$$
\begin{aligned}
\dot{\varepsilon} & =\dot{\varepsilon}_{e}+\dot{\varepsilon}_{p} \\
\dot{\omega} & =\dot{\omega}_{e}+\dot{\omega}_{p}
\end{aligned}
$$

where $\dot{\varepsilon}_{e}$ and $\dot{\omega}_{e}$ (respectively $\dot{\varepsilon}_{p}$ and $\dot{\omega}_{p}$ ) are the elastic strain rate and elastic spin rate (respectively plastic strain rate and plastic spin rate). Thus, the stress rate $\dot{\sigma}$ at point $x$ is given by the following relation:

$$
\dot{\sigma}=c:\left(\dot{\varepsilon}-\dot{\varepsilon}_{p}\right)
$$

\subsection{Constitutive law}

In the present work, the level of discretization is not sufficient to explicitly model the pearlitic microstructure. In other words, each voxel of the discretized microstructure either corresponds to pearlite or to proeutectoid $\alpha$-ferrite. For pearlitic voxels, since two different phases are present over the voxel, it is needed to partition the stress and strain tensors between cementite (denoted by superscript $\mathrm{Fe}_{3} \mathrm{C}$ ) and eutectoid $\alpha$-ferrite (denoted by superscript $\alpha$ ). An upper-bound approximation (i.e. Voigt approximation) is used here so the strain rate and spin rate tensors are considered to be identical in both eutectoid $\alpha$-ferrite and cementite:

$$
\begin{aligned}
\dot{\varepsilon}^{F e_{3} C} & =\dot{\varepsilon}^{\alpha}=\dot{\varepsilon} \\
\dot{\omega}^{F e_{3} C} & =\dot{\omega}^{\alpha}=\dot{\omega}
\end{aligned}
$$

A Hall-Petch type of relationship with respect to the interlamellar spacing is generally observed in pearlite such that eutectoid $\alpha$-ferrite is generally much harder than proeu- 
tectoid $\alpha$-ferrite (Modi et al., 2001). Consequently, as a first assumption, a purely elastic behavior is assumed for both cementite and eutectoid $\alpha$-ferrite in pearlitic voxels:

$$
\begin{gathered}
\dot{\sigma}^{\mathrm{Fe}_{3} \mathrm{C}}=c^{\mathrm{Fe}_{3} \mathrm{C}}: \dot{\varepsilon}^{\mathrm{Fe}_{3} \mathrm{C}} \\
\dot{\sigma}^{\alpha}=c^{\alpha}: \dot{\varepsilon}^{\alpha}
\end{gathered}
$$

The elastic properties of a pearlitic voxel are obtained with the following relation:

$$
c=f c^{F e_{3} C}+(1-f) c^{\alpha}
$$

where $f$ is the volume fraction of cementite in pearlite (i.e. $12 \%$ ).

The proeutectoid ferritic phase is assumed to follow an elasto-viscoplastic behavior. Plastic anisotropy is accounted for by using the crystal plasticity framework. Therefore, if $N$ slip systems (with slip plane normal $n^{g}$ and slip direction $m^{g}$ ) are considered, the plastic strain and spin rates are written as functions of the symmetric and skewsymmetric Schmid tensors $R^{g}$ and $S^{g}$ :

$$
\begin{aligned}
& \dot{\varepsilon}_{p}=\sum_{g=1}^{N} \frac{1}{2}\left(m^{g} \otimes n^{g}+n^{g} \otimes m^{g}\right) \dot{\gamma}_{p}^{g}=\sum_{g=1}^{N} R^{g} \dot{\gamma}_{p}^{g} \\
& \dot{\omega}_{p}=\sum_{g=1}^{N} \frac{1}{2}\left(m^{g} \otimes n^{g}-n^{g} \otimes m^{g}\right) \dot{\gamma}_{p}^{g}=\sum_{g=1}^{N} S^{g} \dot{\gamma}_{p}^{g}
\end{aligned}
$$

where $\dot{\gamma}_{p}^{g}$ is the plastic shear rate on the $g^{t h}$ slip system. The kinetic law on a slip system is in the form (Méric et al., 1991):

$$
\dot{\gamma}_{p}^{g}=\left(\frac{\left\langle\left|\tau^{g}-x^{g}\right|-r^{g}-\tau_{0}\right\rangle}{K}\right)^{n} \operatorname{sign}\left(\tau^{g}-x^{g}\right)
$$

In the above relation, $\tau^{g}$ is the resolved shear stress acting on the $g^{\text {th }}$ slip system, $K$ and $n$ are material parameters, $\tau_{0}$ is the initial critical shear stress from which plastic slip is allowed and $r^{g}$ (respectively $x^{g}$ ) is used for the description of isotropic hardening (respectively kinematic hardening). The resolved shear stress is defined from the stress tensor $\sigma$ at point $x$ by:

$$
\tau^{g}=R^{g}: \sigma
$$

The evolution of $r^{g}$ is given by:

$$
r^{g}=\sum_{h} H^{g h} \sum_{i=1,2}\left(Q_{i}\left(1-\exp \left(-B_{i} p^{h}\right)\right)\right) \text { with } \dot{p}^{h}=\left|\dot{\gamma}_{p}^{h}\right|
$$

where $B_{1}, Q_{1}, B_{2}$ and $Q_{2}$ are material parameters and $H$ is a $N \times N$ matrix describing the interactions between the different slip systems. A two-term isotropic hardening rule is used to correctly describe the cyclic behavior of the studied material: a short hardening stage followed by a softening stage (see section 4.1). The kinematic hardening rule is an Armstrong-Frederick type rule (Armstrong and Frederick, 1966) such that:

$$
\dot{x}^{g}=\alpha \dot{\gamma}_{p}^{g}-\beta x^{g}\left|\dot{\gamma}_{p}^{g}\right|=\alpha \dot{y}^{g}
$$

where $\alpha$ and $\beta$ are material parameters. The evolution laws for $r^{g}, x^{g}$ and $\tau^{g}$ can be obtained in an equivalent manner from the state potential $\psi^{g}$ proposed by Vincent (2008) which is defined for each slip system $g$ : 


$$
\begin{aligned}
\psi^{g}= & \frac{1}{2} \mu^{g}\left(\gamma^{g}-\gamma_{p}^{g}\right)^{2}+\frac{1}{2} \alpha y^{g} y^{g}+H^{g g} \sum_{i=1,2}\left(Q_{i}\left(p^{g}+\frac{1}{B_{i}} \exp \left(-B_{i} p^{g}\right)-\frac{1}{B_{i}}\right)\right) \\
& +p^{g} \sum_{g \neq h} H^{g h} \sum_{i=1,2}\left(Q_{i}\left(1-\exp \left(-B_{i} p^{h}\right)\right)\right)-s^{g} T
\end{aligned}
$$

where $\mu^{g}$ is the elastic shear modulus, $s^{g}$ is the specific entropy and $\gamma^{g}$ is the total shear strain of the slip system $g$. In order to be consistent with the above state potential, which is adopted here, the internal variables are $\gamma_{p}^{g}$ (associated with the driving force for plastic slip $-\tau^{g}=\rho \partial \psi^{g} / \partial \gamma_{p}^{g}$ ), $p^{g}$ (associated with the driving force for isotropic hardening $r^{g}=\rho \partial \psi^{g} / \partial p^{g}$ ) and $y^{g}$ (associated with the driving force for kinematic hardening $\left.x^{g}=\rho \partial \psi^{g} / \partial y^{g}\right)$.

\subsection{Dissipation source and stored energy}

Within the generalized standard material framework (Germain et al., 1983), the equilibrium state of a voxel is defined by the following state variables: the temperature $T$, the total strain $\varepsilon$ and the internal variables describing the microstructural state (i.e. $\gamma_{p}^{g}, p^{g}$ and $\left.y^{g}\right)$. According to the choice of internal variables, the dissipation source $d_{1}$ at point $x$ is:

$$
d_{1}= \begin{cases}0 & \text { for pearlite } \\ \sum_{g} \tau^{g} \dot{\gamma}_{p}^{g}-\sum_{g} r^{g} \dot{p}^{g}-\sum_{g} x^{g} \dot{y}^{g} & \text { for proeutectoid } \alpha \text {-ferrite }\end{cases}
$$

By denoting $\langle z\rangle$ the volume average of the field $z(x)$, the macroscopic dissipation source is given by:

$$
D_{1}=<d_{1}>=<\sum_{g} \tau^{g} \dot{\gamma}_{p}^{g}-\sum_{g} r^{g} \dot{p}^{g}-\sum_{g} x^{g} \dot{y}^{g}>
$$

It should be noticed that the plastic work rate (i.e. $\sum_{g} \tau^{g} \dot{\gamma}_{p}^{g}$ ) is not entirely dissipated into heat. Indeed, since the microstructure is changing during the cyclic loading (i.e. isotropic and kinematic hardening), some part of the plastic work is stored in the material because of the internal stresses that are associated with defects (e.g. dislocations, vacancies). The stored energy evolution rate $p_{s}$ at point $x$ is given by:

$$
p_{s}= \begin{cases}0 & \text { for pearlite } \\ \sigma: \dot{\varepsilon}_{p}-d_{1}=\sum_{g} r^{g} \dot{p}^{g}+\sum_{g} x^{g} \dot{y}^{g} & \text { for proeutectoid } \alpha \text {-ferrite }\end{cases}
$$

At the macroscopic scale, the stored energy evolution rate is not the volume average of $p_{s}$. The macroscopic stored energy evolution rate is defined as follows:

$$
\begin{aligned}
P_{s} & =\Sigma: \dot{E}_{p}-D_{1} \\
& =\Sigma: \dot{E}-\Sigma: \dot{E}_{e}-<\sigma: \dot{\varepsilon}_{p}>+<p_{s}> \\
& =\Sigma: \dot{E}-\Sigma: \dot{E}_{e}-<\sigma: \dot{\varepsilon}>+<\sigma: \dot{\varepsilon}_{e}>+<p_{s}>
\end{aligned}
$$

Making use of the Hill-Mandel lemma allows to further simplify the above relation:

$$
P_{s}=\Sigma: \dot{E}_{e}+<\sigma: \dot{\varepsilon}_{e}>+<p_{s}>
$$




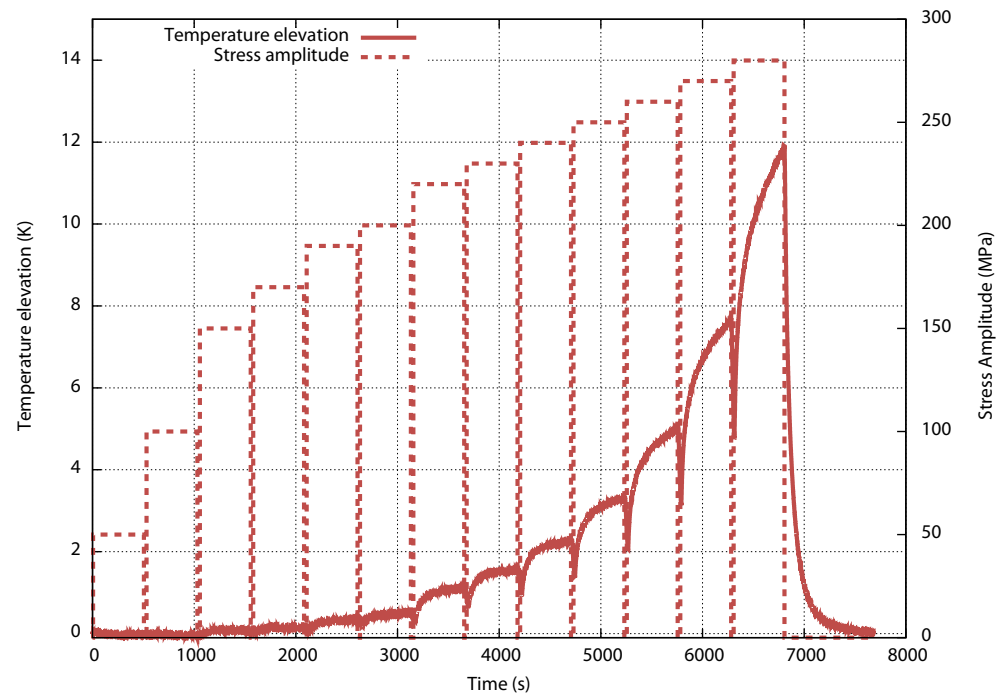

Fig. 2. Evolution of the measured temperature elevation $\bar{\theta}$ and the applied stress amplitude $\Sigma_{a}$ as a function of time.

If the above relation is averaged over one loading cycle, one obtains the expression of the average stored energy evolution rate per cycle:

$$
\bar{P}_{s}=\overline{\left\langle\sigma: \dot{\varepsilon}_{e}>\right.}+\overline{\left\langle p_{s}>\right.}=\overline{\left\langle\sigma: \dot{\varepsilon}_{e}>\right.}+\overline{\left\langle\sum_{g} r^{g} \dot{p}^{g}>\right.}+\overline{\left\langle\sum_{g} x^{g} \dot{y}^{g}>\right.}
$$

Relation (36) shows that energy storage is due to the internal stresses induced by strain incompatibilities between voxels and the internal stresses associated with the presence of defects inside voxels. While the homogenization method explicitly accounts for the internal stresses resulting from strain incompatibilities between voxels, the influence of defects is indirectly described with the phenomenological isotropic and kinematic hardening rules.

\section{Results and discussion}

\subsection{Experimental results}

Two specimens (labeled as EXP1 and EXP2) were submitted to a compression cyclic loading with a constant load ratio $R=\infty$ and a constant frequency $f_{l}=20 \mathrm{~Hz}$. The compression cyclic loading was chosen in order to limit the influence of fatigue damage on dissipative phenomena. The cyclic test was decomposed into several loading blocks of 10000 cyles with increasing stress amplitudes ranging from $50 \mathrm{MPa}$ to $280 \mathrm{MPa}$.

The measured temperature elevation $\bar{\theta}$ and the applied stress amplitude $\Sigma_{a}$ are plotted as a function of time in figure 2. Though a thermal steady state is observed for low stress amplitudes (i.e. $\Sigma_{a}<250 \mathrm{MPa}$ ), it does not exist at high stress amplitudes. The non-existence of a thermal steady state can be explained by a softening mechanism that is sometimes observed in ferritic steels under cyclic loading (Kruml and Polák, 2001; Petrenec et al., 2006). The existence of a softening mechanism is confirmed by 


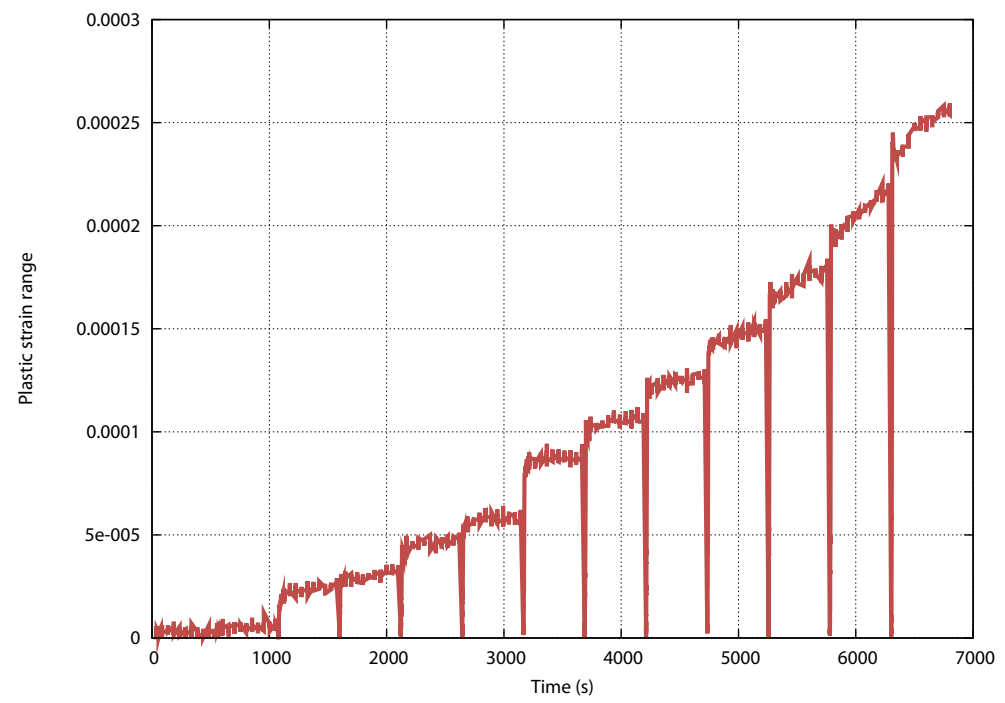

Fig. 3. Evolution of the plastic strain range as a function of the number of cycles.

the observation of the plastic strain amplitude evolution. Indeed, when stress amplitudes are high enough, the plastic strain amplitude continuously increases with the number of applied loading cycles though a short hardening stage is observed for the very first cycles (see figure 3).

Relation (4) has been used to estimate the average dissipated energy per cycle $\bar{W}_{d}=$ $\bar{D}_{1} / f_{l}$ from the measured temperature elevation. The evolution of the average dissipated energy per cycle $\bar{W}_{d}$ (determined for the $1000^{\text {th }}$ cycle) as a function of the stress amplitude $\Sigma_{a}$ is plotted in figure 4 . As already observed by several authors (Berthel et al., 2007; Mareau et al., 2009; Poncelet et al., 2010), the amount of energy which is dissipated into heat during one loading cycle increases with the stress amplitude. Galtier et al. (2002) showed that the dissipated energy augmentation is associated with an increase of the plastic activity that can be correlated with the formation of persistent slip bands.

The average strain energy per cycle $\bar{W}=\bar{P}_{\text {ext }} / f_{l}$, which corresponds to the hysteresis area of the stress-strain curve, has been computed from the stress and strain data for both specimens by using equation (7). The average strain energy per cycle $\bar{W}$ (determined for the $1000^{\text {th }}$ cycle) is plotted as a function of the stress amplitude $\Sigma_{a}$ in figure 5 . The general trend is quite similar to what is observed for dissipated energy: the higher the stress amplitude, the higher the strain energy per cycle. However, no matter the stress amplitude, the strain energy is always superior to the dissipated energy which means that the hysteresis area cannot be identified with the dissipated energy so energy storage cannot be neglected.

The difference between strain and dissipated energies has been used to estimate the average stored energy per cycle $\bar{W}_{s}=\bar{P}_{s} / f_{l}$. Figure 6 represents the fractions of strain energy which are either stored (i.e. $\bar{W}_{s} / \bar{W}$ ) or dissipated into heat (i.e. $\bar{W}_{d} / \bar{W}$ ) as a function of the stress amplitude $\Sigma_{a}$. A significant fraction of the strain energy is stored in the material for low stress amplitudes while most of the strain energy is dissipated into heat for high stress amplitudes. Indeed, most of the strain energy is used for microstructural modifications (e.g. dislocation multiplication) at low stress levels while dissipative 


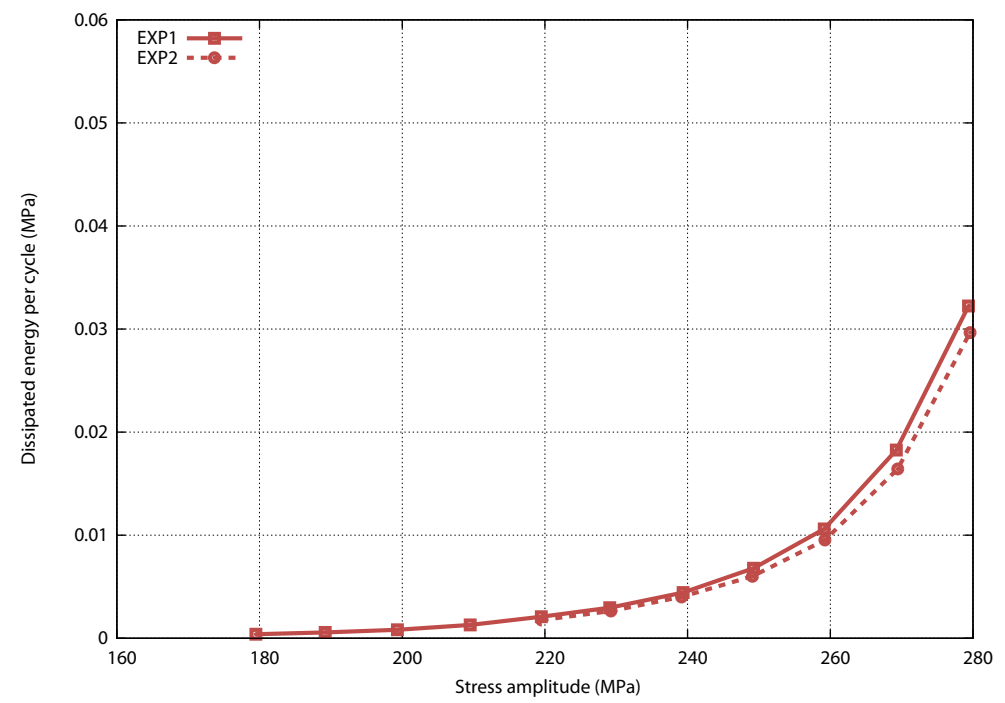

Fig. 4. Evolution of the average dissipated energy per cycle as a function of the applied stress amplitude. The average dissipated energy per cycle has been determined for the $1000^{t h}$ cycle of each block.

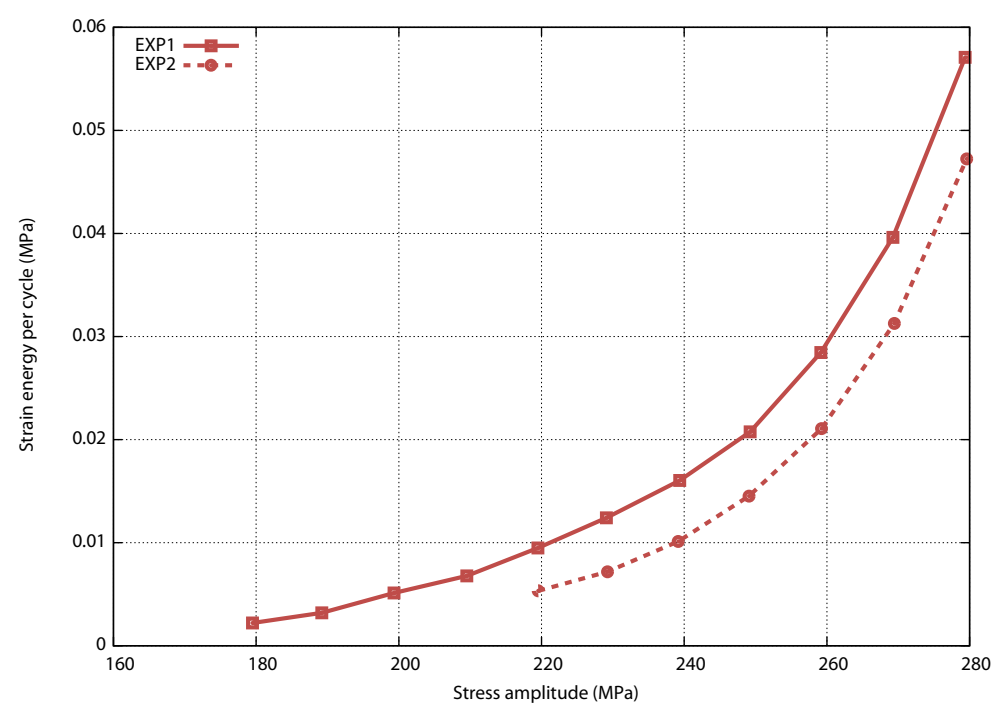

Fig. 5. Evolution of the average strain energy per cycle as a function of the applied stress amplitude. The average strain energy per cycle has been determined for the $1000^{t h}$ cycle of each block.

mechanisms (e.g. dislocation displacements) become predominant at high stress levels. Those results are consistent with the observations of Kaleta (1998) who found stored energy ratios $\bar{W}_{s} / \bar{W}$ of $37.4 \%$ for low cycle fatigue tests and $67.3 \%$ for high cycle fatigue tests for a similar steel grade.

Nevertheless, it should be noticed that the stored and dissipated energy ratios are not constant with respect to time for a given amplitude. As shown in figure 7, when cyclic softening is not significant (i.e. for low stress amplitudes), a stabilized stored energy 


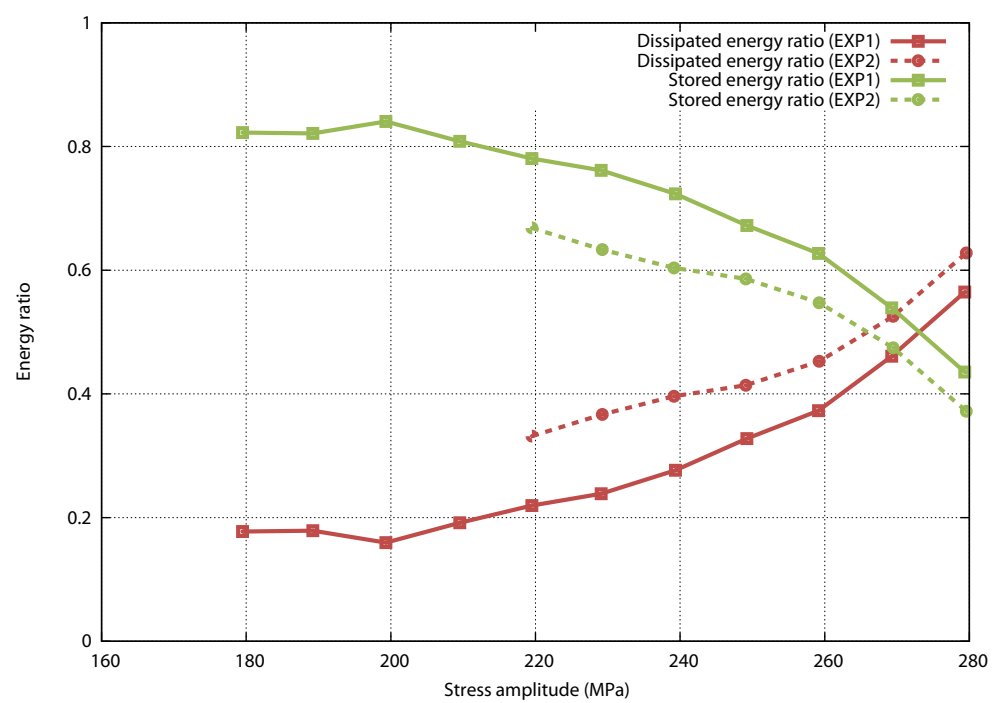

Fig. 6. Evolution of the stored energy and dissipated energy fractions as a function of the stress amplitude. Both ratios have been determined for the $1000^{t h}$ cycle of each block.

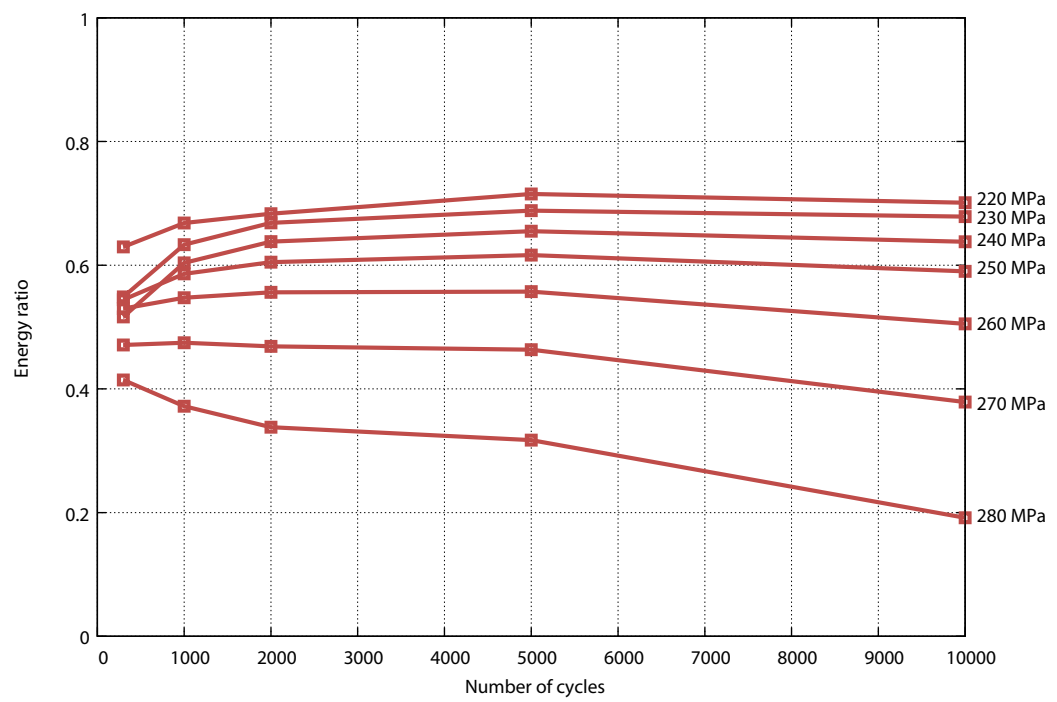

Fig. 7. Evolution of the stored energy fraction as a function of the number of cycles for different stress amplitudes.

ratio is reached after 2000 cycles. At the opposite, when cyclic behavior is influenced by cyclic softening (i.e. for high stress amplitudes), the stored energy ratio significantly decreases after 5000 cycles. Because energy storage is (at least partly) achieved by the multiplication of dislocations, the stored energy ratio diminution is explained by the saturation of the dislocation density that is generally observed in metallic materials (around $10^{16} \mathrm{~m}^{-2}$ for pure $\alpha$-iron (Nakashima et al., 2006)). 


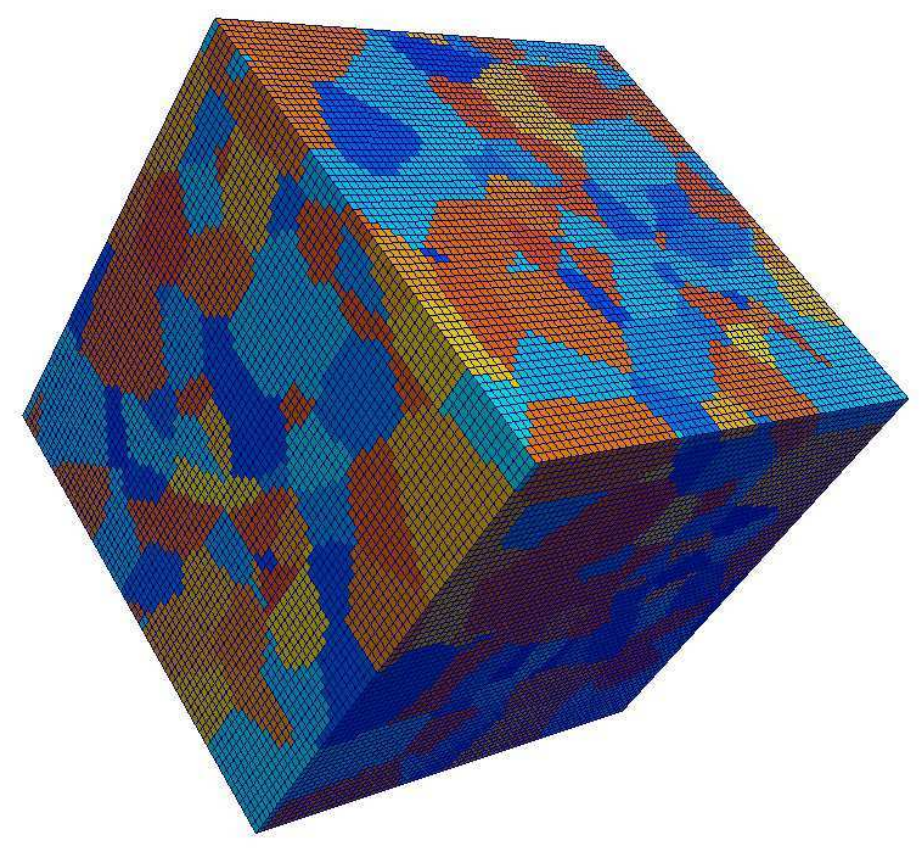

Fig. 8. Representation of the discretized microstructure of the C35 steel grade. Pearlitic voxels appear with a red/orange color while ferritic voxels appear with a blue color.

\subsection{Numerical results}

The model (named SIM1) has been used to obtain estimations of the energy balance at a microscopic scale for a given stress amplitude $\left(\Sigma_{a}=280 \mathrm{MPa}\right)$. The simulations were performed with a polycrystal of 512 grains with orientations representative of the crystallographic texture of the real material. The generation of the polycrystalline aggregate was achieved thanks to the procedure proposed by Robert et al. (2012). The different grains were initially approximated by spheroids being randomly positioned but not superimposed. The Watershed algorithm (Meyer, 1994) was then used to dilate the spheroids to fill the volume element such that smooth grain boundaries were obtained. Finally, as illustrated by figure 8 , the polycrystal was discretized into $64 \times 64 \times 64$ voxels. Both the $\{110\}<111>$ and $\{112\}<111>$ slip systems were considered in the simulations (Berbenni et al., 2004).

The material parameters are given in table 3 . The single crystal elastic constants were taken from Sakata et al. (1990) for $\alpha$-ferrite and from Ledbetter (2010) for cementite. The coefficients of the hardening matrix $H$ were set to 1 for interactions between coplanar systems and 1.1 for interactions between non-coplanar systems (Schmitt et al., 1997). The remaining parameters were identified using only the following experimental data:

- the evolutions of strain, dissipated and stored energies as a function of the number of cycles determined for $\Sigma_{a}=280 \mathrm{MPa}$ for both specimens (i.e. EXP1 and EXP2),

- a cyclic stress-strain curve obtained from a symmetric strain-controlled cyclic test 


\begin{tabular}{|c|c|c|c|c|c|}
\hline$c_{11}^{\alpha}(\mathrm{GPa})$ & $c_{12}^{\alpha}(\mathrm{GPa})$ & $c_{44}^{\alpha}(\mathrm{GPa})$ & $c_{11}^{F e_{3} C}(\mathrm{GPa})$ & $c_{12}^{F e_{3} C}(\mathrm{GPa})$ & $c_{13}^{F e_{3} C}(\mathrm{GPa})$ \\
\hline 232 & 135 & 116 & 394 & 157 & 146 \\
\hline$c_{22}^{F e_{3} C}(\mathrm{GPa})$ & $c_{23}^{F e_{3} C}(\mathrm{GPa})$ & $c_{33}^{F e_{3} C}(\mathrm{GPa})$ & $c_{44}^{F e_{3} C}(\mathrm{GPa})$ & $c_{55}^{F e_{3} C}(\mathrm{GPa})$ & $c_{66}^{F e_{3} C}(\mathrm{GPa})$ \\
\hline 412 & 166 & 360 & 83 & 133 & 136 \\
\hline$Q_{1}(\mathrm{MPa})$ & $B_{1}$ & $Q_{2}(\mathrm{MPa})$ & $B_{2}$ & $\alpha(\mathrm{MPa})$ & $\beta$ \\
\hline 9 & 1000 & -5 & 0.25 & 30000 & 4000 \\
\hline$\tau_{0}(\mathrm{MPa})$ & $K(\mathrm{MPa})$ & $n$ & \multicolumn{3}{|c|}{$H^{g h}$ (coplanar/non-coplanar systems) } \\
\hline 50 & 25 & 10 & \multicolumn{3}{|c|}{$1.0 / 1.1$} \\
\hline
\end{tabular}

Table 3

Material parameters for the C35 steel. Only non-zero elastic constants are indicated.

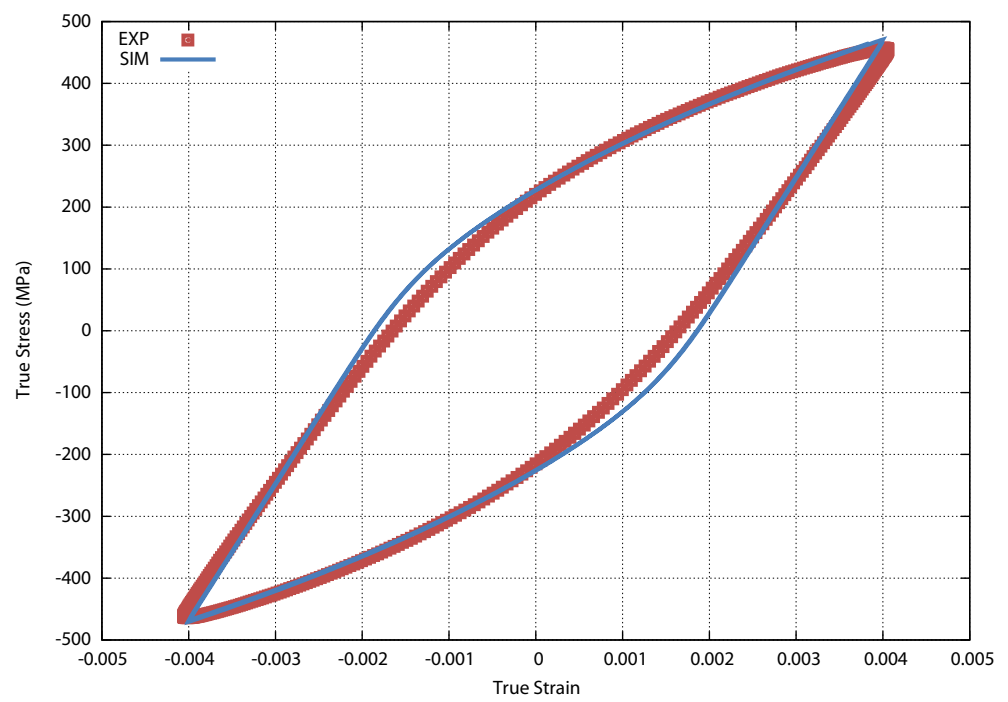

Fig. 9. Experimental (EXP) and calculated (SIM) stress-strain curves obtained from a symmetric straincontrolled cyclic test $( \pm 0.4 \%)$ for the $200^{t h}$ loading cycle.

$( \pm 0.4 \%$ and $1 \mathrm{~Hz})$. The stress and strain data used for parameter identification were obtained during the $200^{\text {th }}$ loading cycle corresponding to a quasi-steady behavior.

A genetic algorithm has been used in order to optimize the selection of parameters to match both the energetic and mechanical behaviors (Skippon et al., 2012). The comparison between the experimental and calculated stress-strain curves is shown in figure 9 for the $200^{\text {th }}$ loading cycle. The experimental and calculated evolutions of the average strain, dissipated and stored energies as a function of the number of cycles are plotted in figure 10.

Though the model provides a reasonable description of the experimental cyclic stressstrain curve, the calculated elastic-plastic transition is too sharp in comparison with the experimental data (see figure 9). Because experimental estimations of dissipated and stored energies were considered for parameter identification, the comparison emphasizes the difficulties for the present model to provide an exact description of both the mechanical behavior and the energetic behavior. Therefore, parameter identification and model 


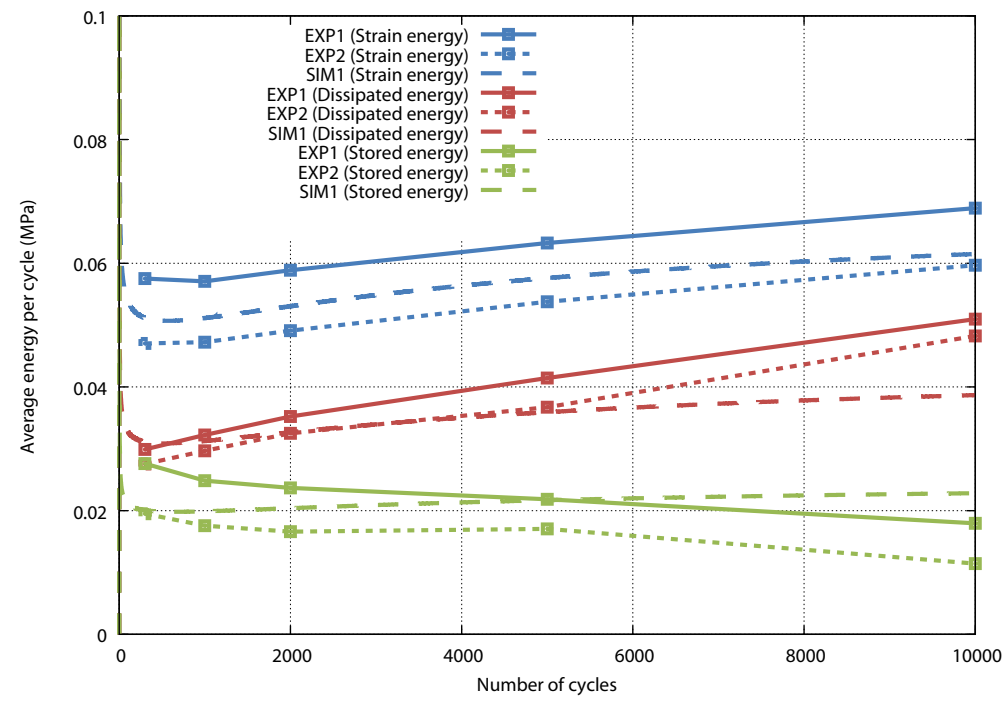

Fig. 10. Experimental and calculated (SIM1) evolutions of the average strain energy, dissipated energy and stored energy per cycle as a function of the number cycles for $\Sigma_{a}=280 \mathrm{MPa}$.

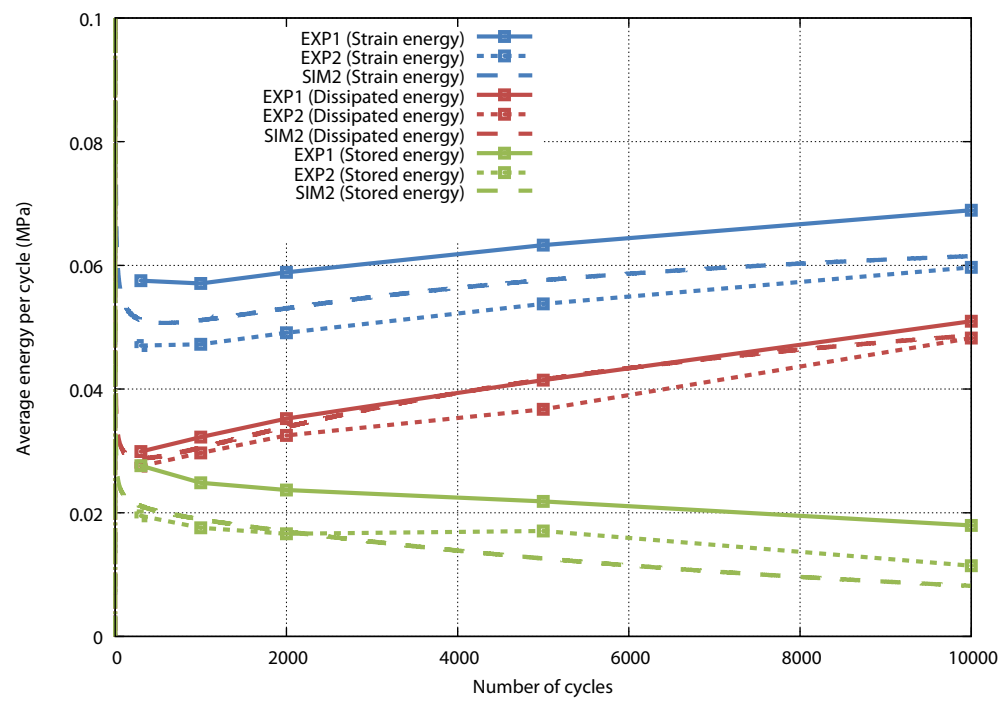

Fig. 11. Experimental and calculated (SIM2) evolutions of the average strain energy, dissipated energy and stored energy per cycle as a function of the number cycles for $\Sigma_{a}=280 \mathrm{MPa}$.

validation could be facilitated by experimental energy estimations since they provide additional information about the material behavior. Also, though a better description could be obtained if supplementary kinematic or isotropic variables were introduced, parameter identification would be tedious with a higher number of model parameters.

Because of cyclic softening, a progressive increase of the average strain energy per cycle, which is correctly captured by the model, is observed with an increasing number of cycles (see figure 10). The model however fails to provide a correct description of the dissipated 


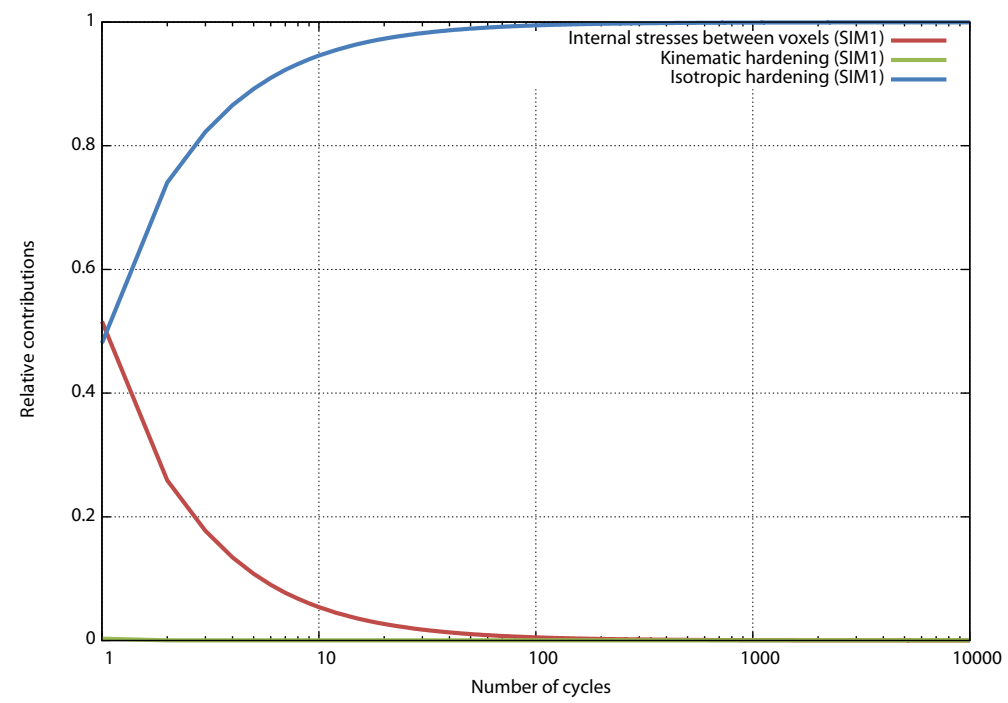

Fig. 12. Relative contributions to the average stored energy per cycle as a function of the number of cycles. The results were obtained from the SIM1 model.

energy and stored energy evolutions beyond 5000 loading cycles. More specifically, while the average stored energy per cycle is experimentally found to decrease with an increasing number of cycles, it increases according to the numerical model. This poor description of the stored energy evolution is due to the definition of the internal variable associated with isotropic hardening. Figure 12 represents the evolution of the relative contributions of the different energy storage mechanisms to the total average stored energy per cycle $\bar{W}_{s}$ as a function of the number of cycles. Isotropic hardening, which accounts for the influence of short-range obstacles such as dislocations, is found to be the main energy storage mechanism. In the present model, energy storage from dislocation multiplication is not bounded since the accumulated shear strain is used as the internal variable associated with isotropic hardening according to the chosen state potential (28). This description is consequently not consistent with experimental observations showing a dislocation density saturation (Nakashima et al., 2006).

The description of the stored energy evolution can be improved if a different choice is made for the internal variable associated with isotropic hardening instead of $p^{g}$. Isotropic hardening can be described from two internal variables $q_{1}^{g}$ and $q_{2}^{g}$ :

$$
q_{i}^{g}=A_{i}\left(1-\exp \left(-M_{i} p^{g}\right)\right) \text { for } i=1,2
$$

In the above relation, $A_{1}, A_{2}, M_{1}$ and $M_{2}$ are material parameters used to describe the diminution which is experimentally observed for stored energy. To keep the evolution law of $r^{g}$ unchanged, the following state potential $\psi^{g}$ is adopted for each slip system $g$ : 


$$
\begin{aligned}
\psi^{g}= & \frac{1}{2} \mu^{g}\left(\gamma^{g}-\gamma_{p}^{g}\right)^{2}+\frac{1}{2} \alpha y^{g} y^{g}+H^{g g} \sum_{i=1,2}\left(A_{i} Q_{i} \frac{M_{i}\left(1-\left(1-\frac{q_{i}^{g}}{A_{i}}\right)^{\frac{B_{i}}{M_{i}}}\right)\left(\frac{q_{i}^{g}}{A_{i}}-1\right)+B_{i} \frac{q_{i}^{g}}{A_{i}}}{M_{i}+B_{i}}\right) \\
& +\sum_{g \neq h} H^{g h} \sum_{i=1,2}\left(A_{i} Q_{i}\left(\frac{q_{i}^{g}}{A_{i}}-1\right)\left(1-\left(1-\frac{q_{i}^{h}}{A_{i}}\right)^{\frac{B_{i}}{M_{i}}}\right)\right)-s^{g} T
\end{aligned}
$$

From the above potential, one can define two driving forces $r_{1}^{g}$ (associated with $q_{1}^{g}$ ) and $r_{2}^{g}$ (associated with $q_{2}^{g}$ ) such that the evolution law of $r^{g}$ is retrieved:

$$
r^{g}=\sum_{i=1,2} r_{i}^{g}=\sum_{i=1,2} \rho \frac{\partial \psi^{g}}{\partial q_{i}^{g}}
$$

This new formulation (labeled as SIM2) requires to identify four additional parameters $\left(A_{1}, A_{2}, M_{1}\right.$ and $\left.M_{2}\right)$. It should be noticed that these parameters do not influence the mechanical behavior so they have to be determined from the experimental estimations of dissipated and stored energies. In the present case, $A_{1}$ and $A_{2}$ were both set to 1.1 and $M_{1}$ and $M_{2}$ were both set to 0.1 . As shown in figure 11, the evolution of the average stored and dissipated energies per cycle as a function of the number of loading cycles is better described with this new thermodynamic formulation. Indeed, an increasing number of cycles corresponds to a decrease of the stored energy ratio. However, this new formulation remains phenomenological and a better understanding of the energy storage and dissipation phenomena would require the explicit modeling of the deformation and hardening mechanisms (e.g. dislocation multiplication, dislocation patterning). The present model could therefore be improved by introducing physically defined internal variables such as in the microstructure sensitive models proposed by Cormier and Cailletaud (2010), Fedelich (2002) and Evrard et al. (2010).

From the SIM2 model it is possible to compute the average dissipated energy and average stored energy per grain. Indeed, the polycrystal is divided according to a $64 \times$ $64 \times 64$ regular grid such that each of the 512 grains is discretized into a few hundred voxels. Thus, the average dissipated energy (or stored energy) for a given grain with $n$ voxels is defined as the weighted average of the local dissipated energy $\bar{w}_{d}$ (or local stored energy $\bar{w}_{s}$ ) computed over the $n$ voxels. When looking at the values of dissipated energy and stored energy in the different grains, the maximum values are found to be about seven times the average values. The main reason for the heterogeneous aspect of the dissipated and stored energy fields is the crystallographic orientation distribution. The dissipated and stored energies are plotted as a function of the maximal Schmid factor for each ferritic grain in figure 13. The dissipated and stored energies are found the highest for grains the most favorably oriented to slip for uniaxial compression according to the Schmid law. This effect, which has already been demonstrated by Mareau et al. (2012) for dissipated energy, is however not sufficient to fully explain the heterogeneous aspect of the energetic fields.

Indeed, as a consequence of the anisotropic single crystal properties, some internal stresses exist in the different grains. Figure 14 represents the dissipated and stored energies as a function of the Von Mises equivalent internal stress for each ferritic grain 


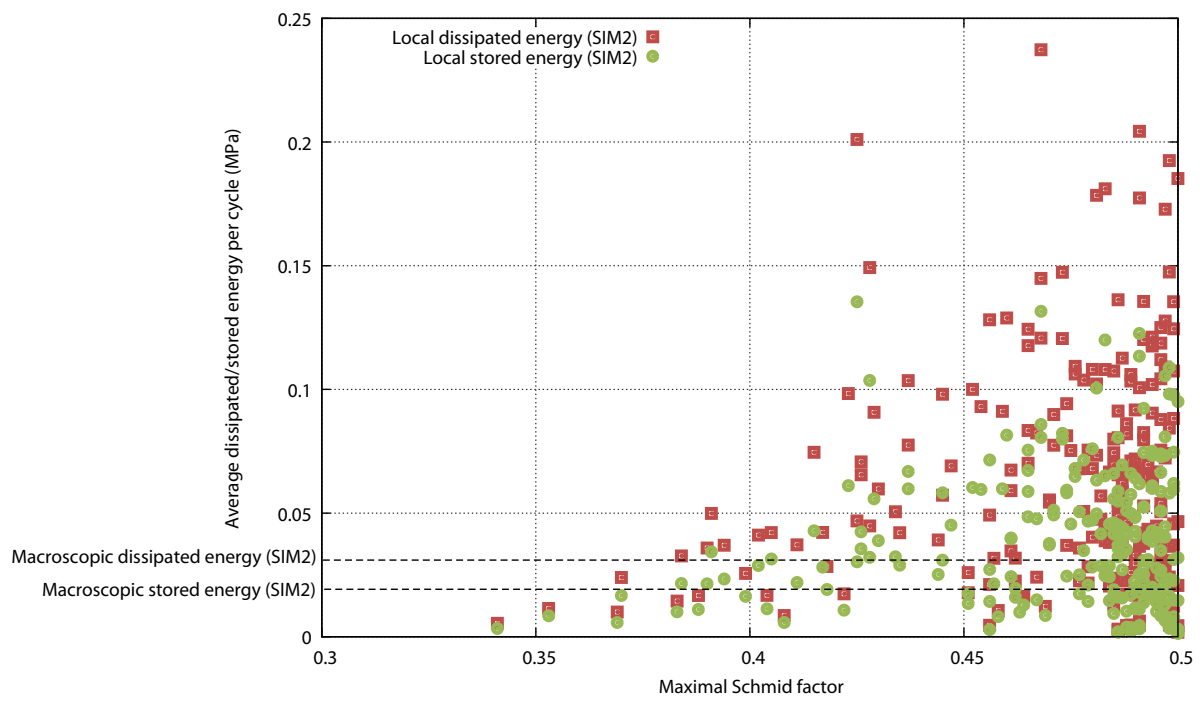

Fig. 13. Average stored energy and dissipated energy per cycle for the different ferritic grains as a function of the maximal Schmid factor. The results were obtained for the $1000^{t h}$ loading cycle for a stress amplitude of $280 \mathrm{MPa}$ with the SIM2 model.

at the end of the $1000^{t h}$ cycle (i.e. in the absence of an external macroscopic stress). Internal stresses are observed to contribute to the heterogeneous character of the stored and dissipated energy fields at the grain level: the higher the internal stresses, the higher the average dissipated and stored energies per cycle. In the present material, the generation of internal stresses during the cyclic loading is facilitated by the presence of a two-phased microstructure since pearlite and proeutectoid $\alpha$-ferrite exhibit very different behaviors. Nevertheless, the impact of the multiphased microstructure on internal stresses is likely to be overestimated by the model since a purely elastic behavior was assumed for pearlite while plastic deformation remains possible. A better description of the role of the multiphased microstructure would require accounting for plastic slip in pearlite. However, parameter identification would then demand to experimentally separate the energetic contributions of each phase so the material parameters associated with proeutectoid $\alpha$-ferrite and pearlite could be determined. In this perspective, the recent experimental progress in the determination of the thermomechanical fields at a local (i.e. grain) scale should help in improving the parameter identification procedure (Seghir et al., 2012).

\section{Concluding remarks}

The energetic behavior of a medium-carbon steel submitted to a uniaxial cyclic loading has been studied. First, an experimental procedure has been followed to obtain experimental estimations of the amount of energy which is either stored or dissipated into heat during one loading cycle. The partition between stored energy and dissipated energy is strongly dependent on the stress amplitude. While most of the strain energy is dissipated into heat for high stress amplitudes, a significant fraction of the strain energy is stored in the material for low stress amplitudes. The identification of dissipated energy with 


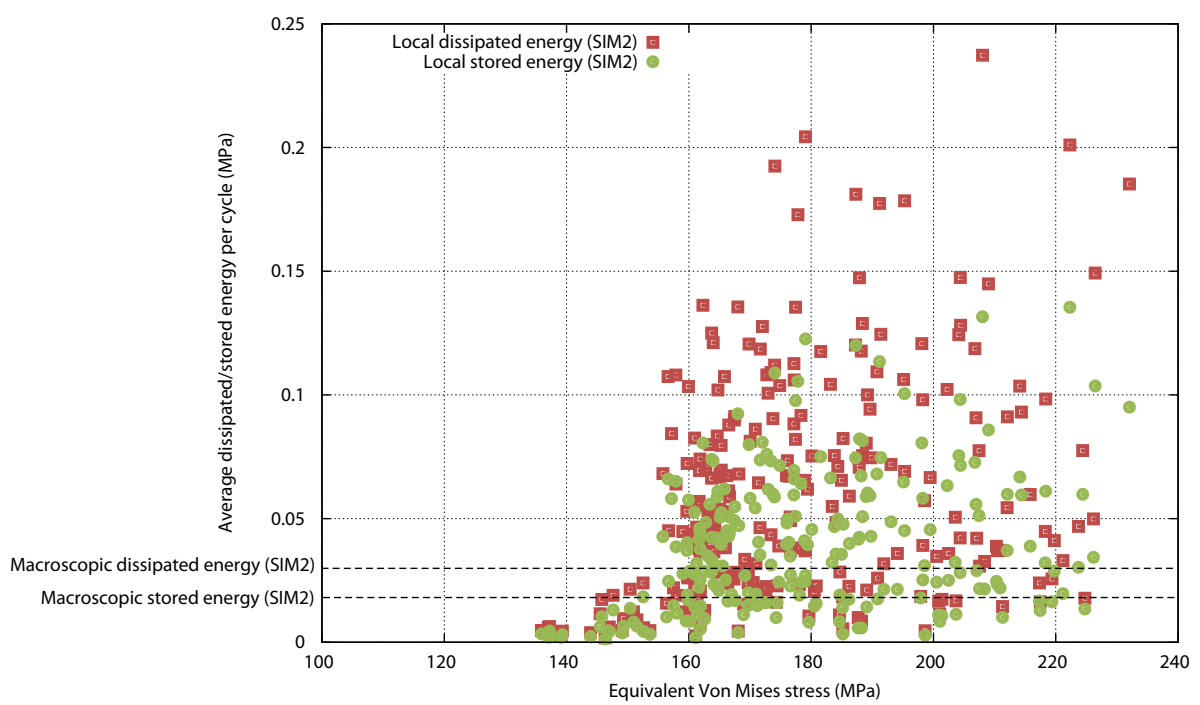

Fig. 14. Average stored energy and dissipated energy per cycle for the different ferritic grains as a function of the Von Mises equivalent stress. The results were obtained for the $1000^{\text {th }}$ loading cycle for a stress amplitude of $280 \mathrm{MPa}$ with the SIM2 model.

strain energy (i.e. the hysteresis area) is therefore not a valid approximation especially for low stress amplitudes. Also, because of the limited capacity of the material for dislocation multiplication, a diminution of the stored energy ratio with an increasing number of cycles is observed for high stress amplitudes.

Then, a numerical model has been developed to gain insight into the energy balance at a microscopic scale. To match the experimental stored energy evolution, a new thermodynamic formulation has been developed. An alternative definition of the internal variables associated with isotropic hardening has been proposed to describe the diminution of the stored energy which is observed when the number of applied cycles increases. Finally, from the results of the model, it has been observed that both the stored energy and dissipated energy fields are strongly scattered. The dispersion is mostly explained by the crystallographic orientation distribution and the two-phased microstructure.

\section{References}

Armstrong, P.J., Frederick, C.O., 1966. A mathematical representation of the multiaxial Baushinger effect, Cegb Report rd/b/n731, Berckeley Nuclear Laboratories.

Banvillet, A., Palin-Luc, T., Lasserre, S., 2003. A volumetric energy based high cycle multiaxial fatigue citerion. International Journal of Fatigue 25, 755-769.

Berbenni, S., Favier, V., Lemoine, X., Berveiller, M., 2004. Micromechanical modeling of the elastic-viscoplastic behavior of polycrystalline steels having different microstructures. Materials Science and Engineering A 372, 128-136.

Berthel, B., Wattrisse, B., Chrysochoos, A., Galtier, A., 2007. Thermographic analysis of fatigue dissipation properties of steel sheets. Strain 43, 273-279.

Berveiller, M., Zaoui, A., 1979. An extension of the self-consistent scheme to plasticallyflowing polycrystals. Journal of the Mechanics and Physics of Solids 26, 325-344. 
Boulanger, T., Chrysochoos, A., Mabru, C., Galtier, A., 2004. Calorimetric analysis of dissipative and thermoelastic effects associated with the fatigue behavior of steels. International Journal of Fatigue 26, 221-229.

Chrysochoos, A., Berthel, B., Latourte, F., Galtier, A., Pagano, S., Wattrisse, B., 2008. Local energy analysis of high-cycle fatigue using digital image correlation and infrared thermography. Journal of Strain Analysis for Engineering Design 43, 411-421.

Cormier, J., Georges Cailletaud, G., 2010. Constitutive modeling of the creep behavior of single crystal superalloys under non-isothermal conditions inducing phase transformations. Materials Science and Engineering A 527, 6300-6312.

Crossland, B., 1956. Proc. Int. Conf. Fatigue of Metals, 138-149.

Cugy, P., Galtier, A., 2002. Microplasticity and temperature increase in low carbon steels. Proc. 8th Int. Fatigue Conf., 549-556.

Dang Van, K., 1973. Sur la résistance à la fatigue des métaux. Sci. Tech. Armement 47.

Déprés, C., Robertson, C.F., Fivel, M.C., 2006. Low-strain fatigue in 316L steel surface grains: A three dimension discrete dislocation dynamics modelling of the early cycles. Part 2: Persistent slip markings and micro-crack nucleation. Philosophical Magazine 86, 79-97.

Ellyin, F., 1974. A criterion for fatigue under multiaxial states of stress. Mechanics Research Communications 1, 219-224.

Evrard, P., Alvarez-Armas, I., Aubin, V., Degallaix, S., 2010. Polycrystalline modeling of the cyclic hardening/softening behavior of an austeniticferritic stainless steel. Mechanics of Materials 42, 395-404.

Eyre, D.J., Milton,G.W., 1999. A fast numerical scheme for computing the response of composites using grid refinement, Journal of Physique III 6, 41-47.

Fedelich, B., 2002. A microstructural model for the monotonic and the cyclic mechanical behavior of single crystals of superalloys at high temperatures. International Journal of Plasticity 18, 1-49.

Galtier, A., Bouaziz, O., Lambert, A., 2002. Influence of steel microstructure on their mechanical properties. Mécanique et Industries 3, 457-462.

Germain, P., Nguyen, Q.S., Suquet, P., 1983. Continuum thermodynamics. Journal of Applied Mechanics 50, 1010-1020.

Hill, R., 1965. Continuum micro-mechanics of elastoplastic polycrystals. J. Mech. Phys. Solids 13, 89-101.

Hutchinson, J.W., 1976. Bounds and self-consistent estimates for creep of polycrystalline materials. Proc. R. Soc. Lond.A 348, 101-127.

Kaleta, J., 1998. Determination of cold work energy in LCF/HCF region. Proceedings of the Fourth International Conference on Low Cycle Fatigue and Elastoplastic Behaviour of Materials, 93-98.

Kruml, T., Polák, J., 2001. Fatigue softening of X10CrAl24 ferritic steel. Materials Science and Engineering A 319-321, 564-568.

Louche, H., Chrysochoos, A., 2001. Thermal and dissipative effects of accompanying lders band propagation. Materials Science and Engineering A 307, 15-22.

Lebensohn, R. A., Kanjarla, A. K., Eisenlohr, P., 2012. An elasto-viscoplastic formulation based on fast fourier transforms for the prediction of micromechanical fields in polycrystalline materials. International Journal of Plasticity 32-33, 59-69.

Lebensohn, R.A., Tomé, C.N., 1993. A self-consistent anisotropic approach for the simulation of plastic deformation and texture development of polycrystals: application to 
zirconium alloys. Acta Metallurgica Et Materialia 41, 2611-2624.

Ledbetter, H., 2010. Polycrystalline elastic constants of in situ cementite (Fe3C). Materials Science and Engineering A 527, 26572661.

Leis, B.N., 1977. An energy-based fatigue and creep-fatigue damage parameter. J. Pressure Vessel Technol. 99, 524.

Lemaitre, J., Chaboche, J.L., Benallal, A., Desmorat, R., 2009. Mécanique des matériaux solides, third ed. Dunod.

Mareau, C., Favier, V., Weber, B., Galtier, A., 2009. Influence of the free surface and the mean stress on the heat dissipation in steels under cyclic loading. International Journal of Fatigue 31, 1407-1412.

Mareau, C., Favier, V., Weber, B., Galtier, A., Berveiller, M., 2012. Micromechanical modeling of the interactions between the microstructure and the dissipative deformation mechanisms in steels under cyclic loading. International Journal of Plasticity 32-33, 106-120.

Marquis, D., 1979. Modélisation et identification de l'écrouissage anisotrope des métaux. Ph.D. Thesis, University Paris 6.

Méric, L., Poubanne, P., Cailletaud, G.,1991. Single crystal modeling for structural calculations. Part 1: model presentation. J. of Eng. Materials and Technology 113, 162-170.

Meyer, F., 1994. Topographic distance and watershed lines. Signal Processing 38 (1), $113-125$.

Michel, J.C., Moulinec, H., Suquet, P., 2001. A computational scheme for linear and non-linear composites with arbitrary phase contrast, Int. J. Numer. Meth. Engng 52, 139-160.

Modi,O.P., Deshmukh, N., Mondal, D.P., Jha, A.K., Yegneswaran, A.H., Khaira, H.K., 2001. Effect of interlamellar spacing on the mechanical properties of $0.65 \% \mathrm{C}$ steel. Materials Characterization 46, 347-352.

Morel, F., 2000. A critical plane approach for life prediction of high cycle fatigue under multiaxial variable amplitude loading. International Journal of Fatigue 22, 101-119.

Moulinec, H., Suquet, P., 1998. A numerical method for computing the overall response of nonlinear composites with complex microstructure. Computer Methods in Applied Mechanics and Engineering 157, 69-94.

Mura, T., 1987. Micromechanics of defects in solids, second ed. Springer.

Mura, T., 1994. A theory of fatigue crack initiation. Materials Science and Engineering A $176,61-70$.

Nakashima, K., Suzuki, M., Futamura, Y., Tsuchiyama, T., Takaki, S., 2006. Limit of dislocation density and dislocation strengthening in iron. Materials Science Forum 503504, 627-632.

Palin-Luc, T., Lasserre, S., 1998. An energy based criterion for high cycle multiaxial fatigue. European Journal of Mechanics 17, 237-251.

Papadopoulos, I.V., 1994. A new criterion of fatigue strength for out-of-phase bending and torsion of hard metals. International Journal of Fatigue, 16, 377-384.

Paquin, A., Berbenni, S., Favier, V., Lemoine, X., Berveiller, M., 2001. Micromechanical modeling of the elastic-viscoplastic behavior of polycrystalline steels. International Journal of Plasticity 17, 1267-1302.

Petrenec, M., Polák, J., Obrtlík, K., Man, J., 2006. Dislocation structures in cyclically strained X10CrAl24 ferritic steel. Acta Materialia 54, 3429-3443.

Polák, J., 2007. Mechanisms and kinetics of the early fatigue damage in crystalline ma- 
terials. Materials Science and Engineering A 468-470, 33-39.

Poncelet, M., Doudard, C., Calloch, S., Weber, B., Hild, F., 2010. Probabilistic multiscale models and measurements of self-heating under multiaxial high cycle fatigue. Journal of the Mechanics and Physics of Solids 58, 578-593.

Robert, C., Saintier, N., Palin-Luc, T., Morel, F., 2012. Micro-mechanical modelling of high cycle fatigue behaviour of metals under multiaxial loads. Mechanics of Materials $55,112-129$.

Roters, F., Eisenlohr, P., Hantcherli, L., Tjahjanto, D. D., Bieler, T. R., Raabe, D., 2010. Overview of constitutive laws, kinematics, homogenization and multiscale methods in crystal plasticity finite-element modeling: Theory, experiments, applications. Acta Materialia 58, 1152-1211.

Sakata, K., Daniel, D., Jonas, J.J., 1990. Estimation of 8th, 10th and 12th order odf coefficients from elastic properties in cold rolled steel sheets by adjustment of single crystal elastic constants. Textures and Microstructures 12, 175-185.

Schmitt, C., Lipinski, P., Berveiller, M., 1997. Micromechanical modelling of the elastoplastic behavior of polycrystals containing precipitates-application to hypo- and hypereutectoid steels. International Journal of Plasticity 13, 183-199.

Seghir, R., Bodelot, L., Charkaluk, E., Dufrénoy, P., 2012. Numerical and experimental estimation of thermomechanical fields heterogeneity at the grain scale of 316L stainless steel. Computational Materials Science 53, 464-473.

Sines, G., 1959. Metal Fatigue, McGraw Hill, New York.

Skippon, T., Mareau, C., Daymond, M.R., 2012. On the determination of single-crystal plasticity parameters by diffraction: optimization of a polycrystalline plasticity model using a genetic algorithm. J. Appl. Cryst. 45, 627-643.

Vincent, L., 2008. On the ability of some cyclic plasticity models to predict the evolution of stored energy in a type 304L stainless steel submitted to high cycle fatigue. European Journal of Mechanics 27, 161-180.

Warren, J., Wei, D.Y., 2010. A microscopic stored energy approach to generalize fatigue life stress ratios. International Journal of Fatigue 32, 1853-1861.

Zehnder, A.T., 1991. A model for the heating due to plastic work. Mechanics Research Communications 18, 23-28.

Zeman, J., Vondrejc, J., Novák, J., Marek, I., 2010. Accelerating a FFT-based solver for numerical homogenization of periodic media by conjugate gradients. Journal of Computational Physics 229, 8065-8071. 